\title{
Source-Normalized Impact per Paper (SNIP)
}

Bo-Christer Björk, David Solomon

\section{Source}

Bo-Christer Björk and David Solomon. (2014). Developing an Effective Market for Open Access Article Processing Charges (PDF) (Report). Wellcome Trust.

SNIPS are similar to impact factors listed in the Journal Citation Reports, but calculated using citation data from Scopus. The impact factors are normalized around one for each discipline to neutralize the effects of differences in citation practices across different disciplines. 